\title{
Study On HIV-1 Drug Resistance
}

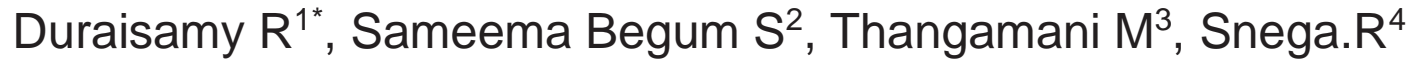 \\ ${ }^{1}$ Professor, Department of Pharmacognosy, Nandha College of Pharmacy, Erode, \\ Tamilnadu, Email:duraisami@nandhapharmacy.org \\ ${ }^{2}$ Assistant Professor, Department of Pharmacognosy, Nandha College of Pharmacy, \\ Erode, Tamilnadu, Email:jesirajawidh@gmail.com \\ ${ }^{3}$ Assistant Professor, Department of Pharmacognosy, Nandha College of Pharmacy, \\ Erode, Tamilnadu, Email: thangamani@nandhapharmacy.org \\ ${ }^{4}$ Assistant Professor, Department of Community Health Nursing, Nandha College of \\ Nursing, Erode, Tamilnadu, Email: snegariya@gmail.com \\ ${ }^{*}$ Corresponding Author
}

\begin{abstract}
Drug tolerance is an important element in analyzing the characteristics of any illness. The goal of this paper is to explain the distinctions between HIV-1 patient groups and virus subtypes in terms of disease progression, result of treatment and production of drug resistance. It also seeks to identify the antiretroviral use and drug resistance growth of both HIV-1 subtype and recombinant modes of therapy with improvements and temporal patterns.
\end{abstract}

Keywords: Drug Resistance, HIV-1, Therapy

\section{Introduction}

Regardless of whether new prescriptions are created, drug obstruction stays an issue. Alongside the high replication pace of the infection, mistake inclined obstruction encourages the creation of medication opposition checking if replication isn't completely forestalled by fruitful ART. A multitude of various hereditary changes, purported viral quasis living beings, is the viral populace among tainted patients. In any case, the replicative capacity of the transformed semi species is regularly not as effective as that of the infection of the wild structure. Thusly, if meds are not, at this point in the framework, wildtype structures win. Medication resilience screening with no impact on viral wellbeing will last more and is more powerless against relational scattering. How quickly and successfully drug opposition observing develops relies upon numerous variables that are additionally basic to the supposed hereditary limit of the medication. Both helped Pls have a high hereditary obstruction and, in spite of proceeding with replication during treatment disappointment, changes are delayed to develop. There are two significant NRTI opposition components; one is the RT protein alteration that encourages it to separate among NRTIs and simple substrates, prompting a diminished combination of NRTIs into the DNA chain. The NRTI embedded into the viral DNA is taken out by the other interaction. Thymidine simple freaks are known as NRTIs (TAMs). There are additionally unique TAM pathways, which cause more noteworthy protection from NRTI. In the protein pocket where NNRTIs tie, NNRTIs tie to HIV-1 RT and NNRTI transformations are discovered, hence decreasing the proclivity of the NNRTIs to the compound. Medication resilience happens quickly if the HIV-1 replication isn't completely hushed or if the NNRTI is utilized as monotherapy. The fast rise of Drug Resistance Monitering is because of the assortment inside a living being of previous freak infections. Studies have shown that for NNRTI Opioid Resistance Monitering, NVP utilized as single portion treatment in mother-tokid anticipation in LMICs can be picked.

Antiretroviral meds can be ordered by the progression of the existence pattern of HIV-1, which is obstructed by the drug. HIV-1 is an encompassed infection with the heart having two strands of ribonucleic corrosive (RNA). Chemicals that are compulsory for viral replication are additionally present in the viral spot. Switch transcriptase (RT), integrase (IN), and protease are the accompanying proteins: (PR). There are presently four sorts of antiretroviral drugs expected to impede these chemicals, and half breed arrangements have additionally been delivered in a solitary pill design containing at least two medications. HIV-1 replication requires different advances; the relationship between the CD4 receptor (on the host cell) and the two glycoproteins gp120 and gp41 in the infection envelope is at first shaped. The infection at that point ties to the CCR5 or CXCR4 coreceptors, bringing about a move in compliance that empowers the combination of the phone layer and the 
infection. Inhibitors of passage or combination block the limiting, combination as well as section into the host cell of HIV-1. The integraase catalyst catalyzes the fuse of viral DNA into the genome of the host cell. When the infection's DNA is coordinated into the host genome, it is recreated along with the host cell's genome. Three separate Integrase Inhibitors (INI) have been authorized for use. To produce the parts of the develop protein required for HIV-1 to be irresistible, the movement of the PR compound is fundamental. In the last phase of HIV-1 replication, the PR divides the enormous viral forerunner polypeptide chain into proficient proteins. The protease chemical is inactivated by the six protease inhibitors (PI) at present enlisted, and the recently integrated virions are consequently non-irresistible.

\section{Resistance to inhibitors}

By restricting to the PR's dynamic site, Pls evade cleavage of the two proteins. Youthful particles of non-irresistible infections are along these lines delivered. At the substrate-restricting separated of the PR, essential changes are situated and meddle with the limiting of the PI to the PR. Essential transformations alone may littly affect PI opposition and frequently lead to diminished infection wellness. Notwithstanding, when patients proceed with their treatment, auxiliary changes arise that lead to undeniable level opposition and expanded wellness alongside the essential transformations. Between section inhibitors and other antiretroviral drugs, the opposition pathways contrast notably. Combination inhibitors smother HIV-1 and CD4+ T-cell film combination interceded by gp41 and co-receptor inhibitors block the limiting of gp120 to the CCR5 or CXCR4 co-receptors. Changes in an area including 10 amino acids between position 36 and 45 in the HR1 district of gp41 are a focal instrument of protection from combination inhibitors. INI, usually alluded to as integrase inhibitor obstruction, develops rapidly at the hour of viral disappointment. The advancement of novel essential INI Drug Resistance Monitering is trailed by optional transformations which further diminish weakness or potentially increment viral wellness.

\section{HIV Drug Resistance}

Initially, the drawback of obtained and circulated drug opposition was kept to asset rich countries. The point has spread to different nations since the globalization of ART. The appraisal of medication obstruction in asset restricted nations isn't accessible for typical clinical practice. Sentinel destinations were rather utilized for populace level reconnaissance of both obtained and circulated drug opposition. With an improvement in sent NNRTI opposition because of the public scale-up of ARV. In Latin America/Caribbean, North America, and upperpay Asian nations, NNRTI related TDRs have likewise expanded. Around $70 \%$ of viral disappointment patients with NNRTI have NRTI/NNRTI drug opposition transformations in view of the low hereditary boundary. In clinical practice, the shortfall of ordinary viral burden testing will bring about long stretches of virological disappointment before treatment improves. Expansions in both obtained and dispersed medication obstruction will hypothetically impact the adequacy of ART around the world because of development and travel. HIV-1 and HIV-2 and other HIV-1 heredities have brought about numerous zoonotic transmissions from primates to people. In gathering $\mathrm{AH}, \mathrm{HIV}-1$ is arranged into four gatherings: $M, N, O, P$ and HIV-2. Not exclusively does the extreme heterogeneity of the infection help the advancement of medication obstruction control, yet it has additionally added to the improvement of numerous subtypes and recombinants of HIV-1. The essential driver of the worldwide pandemic is HIV-1 gathering $M$ (Major), which has enhanced into extra hereditary strains. In view of the locales contemplated and the subtypes, contrasts in the nucleotide level may exist inside the different subtypes. It has additionally been appeared by complete genome sequencing that blends are typical between subtypes. Coursing recombinant structures (CRF) are alluded to as recombinant infections inside various HIV-1 $M$ subtypes if present in at least three people with various birthplaces of HIV-1M disease. On the off chance that they are absent in at least three individuals, extraordinary recombinant variations are called (URF). With unmistakable local patterns, subtypes got from specific districts are spread. HIV-1 variety may affect sickness movement, spread, advancement of medication obstruction, conclusion, control of viral burden, safe reaction and improvement of antibodies.

Portability and movement to other topographical areas at populace level and transmission between hazard bunches are quickly advancing and new subpandemics and recombinant variations of HIV-1 are being recognized. As they are as unmistakable from one another as they are from HIV-1B, pooling non- $B$ subtypes as a special hereditary gathering ought to be dodged. The phenotypic highlights of the PR and RT catalysts, phenotypic affectability to antiretroviral drugs, and the advancement of subtype-explicit genotypic examples of medication resistance impact contrasts in the pol-quality between subtypes. Viral protease is the objective of protease inhibitors and is as of now the second-line treatment for LMICs where HIV-1C prevails. Contrasts in medication obstruction among subtypes can likely be clarified by PR and Resistance Treatment's enzymatic capacity. Medication Resistance Monitering can prompt expanded or diminished reactant action or may debilitate understanding obstruction restraint or adjustment. Gem structures are relied upon to analyze modified enzymatic properties at the subatomic degree of safe aggregates. The protease is a dimer and there are 99 amino acids in every monomer . In the protease where the protease inhibitors tie, 
the fold is a flexible design. The pivot territory, which is inseparable from development and adjustment of the fold, is close to the fold. To permit substrate that ought to be catalyzed to enter and tie, the fold unit should be adaptable and attempt developments. It is additionally important for the folds to be adaptable, yet diminished substrate adaptability and diminished restricting of a protease inhibitor are the potential ramifications of an excess of adaptability. Gem structures are required to inspect adjusted enzymatic properties at the atomic degree of safe aggregates. The Los Alamos HIV-1 successions are contained in the agreement groupings for various subtypes.

\section{HIV subtyping}

The ViroSeqTM HIV-1 Genotyping System made populace based arrangements of the pol quality, including districts encoding ResistanceTherapy, protease, and integrase, and entered the HIV information base. Critical Drug Resistance Control was set up utilizing the Stanford HIV db calculation and the degree of clinically important opposition for each medication was determined. The most recent available Stanford HIVdb calculation was utilized at the hour of the examination. Rendition 3 REGA subtyping strategy (REGA v3), which utilizes a refreshed choice tree calculation and has better affectability for unadulterated subtype and recombinant discovery. Utilizing the bootscan investigation actualized in the Rega v3 strategy and tried in the SimPlot v3 program, the URFs were additionally characterized. Changing patients from first to second-line In both tried endurance percentiles, ART with virologic disappointment however with no Drug Resistance Management or with virologic disappointment and Drug Resistance Management, exhibited a significantly negative hole so as to second-line VF comparative with patients exchanging without disappointment. A fascinating finding with regards to this exploration, in any case, was that if infection disappointment occurs and drug opposition control is identified, treatment disappointment is controlled effectively in current clinical practice on the grounds that the type of switch and VL didn't show any considerable effect on middle CD4+ T-cell checks at 12 and two years, AIDS time or passing at second-line ART commencement. For some low-pay nations that don't utilize viral burden checking, this finding additionally has results since it features the estimation of viral burden observing in nations where its utilization isn't important for the norm of treatment. The consideration change will be followed effectively if viral burden observation is presented, which would improve the wellbeing status of HIV-1 tainted patients.

\section{Conclusion}

This work gives an examination of the results of the
HIV-1 flare-up and antiretroviral treatment, remembering upgrades for the utilization of ART, elements of medication obstruction control and spread of subtypes over the long haul. It shows how travel and relocation are driving movements in Sweden and the nation is in this way a piece of the worldwide scourge. The considerably more typical clarifications for moving from first-line ART are harmfulness as well as straightforwardness. There is a raised possibility of viral misfortune while switching back and forth among first and second line ARTs if the patient has a high VL. Notwithstanding, the CD4 T-cell check doesn't have a significant impact. Patients contaminated with HIV-1 have a more prominent possibility of infection disease because of protease inhibitors. The normally happening polymorphisms in the HIV-1 protease that may disable the limiting might be a potential contributing reason to the expanded danger of viral disappointment among HIV-1 contaminated patients. Subtype one of a kind long haul tolerant reactions to various antiretroviral drugs, all things considered, associates ought to be tended to while finishing up by far most of the worldwide HIV trouble.

\section{Reference}

1. Dauber DS, Ziermann R, Parkin N, Maly DJ, Mahrus S, Harris JL, et al. Altered substrate specificity of drugresistant human immunodeficiency virus type 1 protease. J Virol 2002,76:1359-1368.

2. Xie D, Gulnik S, Gustchina E, Yu B, Shao W, Qoronfleh $W$, et al. Drug resistance mutations can effect dimer stability of HIV-1 protease at neutral $\mathrm{pH}$. Protein Sci 1999,8:1702-1707.

3. Pieniazek D, Rayfield M, Hu DJ, Nkengasong J, Wiktor $\mathrm{SZ}$, Downing $\mathrm{R}$, et al. Protease sequences from HIV-1 group $M$ subtypes $\mathrm{A}-\mathrm{H}$ reveal distinct amino acid mutation patterns associated with protease resistance in protease inhibitor-naive individuals worldwide. HIV Variant Working Group. AIDS 2000,14:1489-1495.

4. Vergne L, Peeters M, Mpoudi-Ngole E, Bourgeois A, Liegeois F, Toure-Kane C, et al. Genetic Diversity of Protease and Reverse Transcriptase Sequences in NonSubtype-B Human Immunodeficiency Virus Type 1 Strains: Evidence of Many Minor Drug Resistance Mutations in Treatment-Naive Patients. Journal of Clinical Microbiology 2000,38:3919.

5. Kiwanuka N, Laeyendecker O, Quinn TC, Wawer MJ, Shepherd J, Robb M, et al. HIV-1 subtypes and differences in heterosexual HIV transmission among HIVdiscordant couples in Rakai, Uganda. AIDS 2009,23:2479-2484.

6. Kantor R. Impact of HIV-1 pol diversity on drug resistance and its clinical implications. Curr Opin Infect Dis 2006,19:594-606.

7. Kantor R, Katzenstein D. Polymorphism in HIV-1 nonsubtype $B$ protease and reverse transcriptase and its potential impact on drug susceptibility and drug resistance evolution. AIDS Rev 2003,5:25-35.

8. Kosakovsky Pond SL, Smith DM. Are all subtypes created equal? The effectiveness of antiretroviral 
therapy against non-subtype B HIV-1. Clin Infect Dis 2009,48:1306- 1309.

9. Rouet F, Foulongne V, Viljoen J, Steegen K, Becquart $P$, Valea D, et al. Comparison of the Generic HIV Viral Load assay with the Amplicor HIV-1 monitor v1.5 and Nuclisens HIV-1 EasyQ v1.2 techniques for plasma HIV1 RNA quantitation of nonB subtypes: the Kesho Bora preparatory study. J Virol Methods 2010,163:253-257.

10. Borman AM, Paulous S, Clavel F. Resistance of human immunodeficiency virus type 1 to protease inhibitors: selection of resistance mutations in the presence and absence of the drug. J Gen Virol 1996,77 ( Pt 3):419426. 